Karina Conde ${ }^{1}$

Aldana Lichtenberger ${ }^{2}$

Raquel Inés Peltzer ${ }^{3}$

Mariana Cremonte ${ }^{4}$

1,2 Becaria Posdoctoral -IPSIBAT-UNMdP-CONICET Argentina

${ }^{3}$ PSIBAT-UNMdP-CONICETProfesora UNMdP Argentina ${ }^{4}$ Investigadora Independiente

ONICET PSIBAT-UNMdPCONICET- Profesora UNMdP Argentina

Trabajo recibido: 04 de Abril de 2017. Aprobado: $01 \mathrm{de}$ Agosto de 2017.

\section{CONSUMO EXCESIVO EPISÓDICO DE ALCOHOL: CAMBIO NATURAL EN ESTUDIANTES UNIVERSITARIOS.}

BINGE DRINKING: NATURAL CHANGE IN UNIVERSITY STUDENTS.

CONSUMO EXCESSIVO EPISÓDICO DE ÁLCOOL: MUDANÇA NATURAL EM ESTUDANTES UNIVERSITÁRIOS.

\section{Resumen}

El consumo abusivo de alcohol puede ser abandonado sin ayuda formal, sin embargo, la información sobre este hecho es escasa, especialmente en jóvenes que adoptan este tipo de consumo. El objetivo de este estudio es estimar la prevalencia de cambio natural del consumo excesivo episódico de alcohol y explorar su relación con consecuencias adversas del consumo y otras características individuales en jóvenes universitarios. En una muestra aleatoria de 828 estudiantes se evaluó el consumo excesivo episódico pasado/actual, problemas relacionados con el consumo de alcohol, uso de otras sustancias psicoactivas, género y estado civil. La prevalencia de cambio natural fue de $7 \%$. El cambio en el consumo excesivo episódico implicó menor probabilidad de problemas psíquicos, episodios de amnesia anterógrada, lesiones, consumo de otras sustancias y una mayor probabilidad de estar en pareja. Se discute el rol de la severidad de las consecuencias en el cambio natural del consumo, posibles factores de protección y potenciales intervenciones.

Palabras clave: consumo de alcohol, reducción del riesgo, Argentina. 


\section{Abstract}

Binge drinking can be quit without formal help; however, there is not enough information on this fact, especially in young people who adopt this kind of consumption. The objective of this study is to estimate the prevalence of natural change regarding binge drinking and explore its relationship with alcohol related problems and other individual characteristics in university students. In a random sample of 828 students, we assessed past or current binge drinking, alcohol related problems, use of other psychoactive substances, gender and marital status. The prevalence of natural change reached $7 \%$. Quiting binge drinking implied a smaller probability of psychic problems, alcohol induced blackouts, injuries and consumption of other substances as well as a greater probability of finding a partner. The role of the severity of the consequences in the natural change in consumption, possible protection factors and potential interventions are discussed.

Key Words: Alcohol consumption, Risk reduction, Argentina

\section{Resumo:}

O consumo abusivo de álcool pode ser abandonado sem ajuda formal, contudo, a informação sobre esse fato é escassa, especialmente em jovens que adotam esse tipo de consumo. O intuito deste estudo é estimar a prevalência da mudança natural no consumo episódico de álcool excessivo e explorar sua relação com consequências adversas do consumo e outras características individuais entre estudantes universitários. Uma amostra aleatória de 828 alunos avaliou o excesso de uso episódico passado / presente, problemas relacionados ao consumo de álcool, uso de outras substâncias psicoativas, gênero e estado civil. A prevalência da mudança natural foi de $7 \%$. A mudança no consumo excessivo episódico implicou uma menor probabilidade de problemas psíquicos, episódios de amnésia anterógrada, lesões, consumo de outras substâncias e uma maior probabilidade de ter parelha. Discute-se o papel da gravidade das consequências na mudança natural de consumo, potenciais fatores de proteção e potenciais intervenções.

Palavras-chave: consumo de álcool, redução de risco, Argentina.

\section{Introducción}

Las conductas que las personas tienen en torno al cuidado de su salud constituyen una preocupación actual en el ámbito de la psicología. A través de los años, distintas teorías han intentado comprender factores asociados a los comportamientos de salud o de riesgo y aquellos que se relacionan con el mantenimiento o cambio de dichas conductas. Uno de los factores de riesgo asociado a cientos de enfermedades y a conductas de riesgo que derivan en suicidios, violencia, y lesiones no intencionales por causas externas (e.g. choques, golpes, quemaduras), es el consumo de alcohol [1].

Las investigaciones indican que el consumo abusivo de alcohol (aquel que excede al consumo moderado o social) es frecuentemente abandonado sin ayuda formal, pero poco se sabe de este proceso y en algunos campos la información es casi inexistente [2]. Estos estudios se han enfocado en los factores relacionados a los comportamientos de riesgo en adultos con trastornos por uso de alcohol, no obstante, es en la juventud dónde comienzan a aparecer los primeros problemas por uso de alcohol [3], por lo que la información en esta población tiene un especial valor preventivo. A nivel nacional e internacional, los jóvenes presentan patrones de consumo, como excesivo episódico, que derivan en consecuencias para la salud como la intoxicación, y en los problemas de salud pública consecuentes $[4,5]$. Este patrón se define como la ingesta de alcohol que se corresponde con cinco o más unidades estándar en el varón, y cuatro o más en la mujer en una ocasión [6]. A pesar de ser una preocupación a nivel mundial, sólo algunos países han investigado al consumo 
excesivo episódico de alcohol en profundidad [7].

Entre los jóvenes, los estudiantes universitarios se encuentran en alto riesgo de morbimortalidad por sus conductas de consumo de alcohol. El hecho de que este grupo presente menor probabilidad de buscar ayuda [9] y una menor disposición a reconocer que la necesitan [10], agrava la situación. En la Universidad Nacional de Mar del Plata, un estudio piloto con estudiantes de Psicología reveló que más de tres de cada 10 estudiantes presentaban consumo excesivo episódico [11]. En otros contextos, se ha observado que el consumo de alcohol aumenta en los primeros años de la universidad. Dicho aumento sería mayor en los varones universitarios [12], aunque en los últimos años se ha señalado que los patrones de consumo se están homogeneizando para ambos sexos [13, 14].

Hay características que podrían estar relacionadas con que la persona no sea capaz de abandonar un tipo de consumo, como excesivo episódico, sin ayuda. Por ejemplo, problemas psíquicos como la depresión o consecuencias negativas como los episodios de amnesia anterógrada inducidos por el alcohol (i. e. episodios en los cuales las personas pueden realizar acciones sin poder recordarlas al día siguiente) indican mayor severidad de problemas relacionados con el alcohol $[11,16,17]$, dificultando posiblemente que la persona abandone el consumo excesivo episódico sin ayuda. También se ha encontrado que los jóvenes que consumen según patrones de riesgo tienen a utilizar múltiples de sustancias psicoactivas, lo que suele derivar en un deterioro mayor [18]. Sin embargo, también a medida que las personas avanzan en la vida adulta, el consumo de alcohol disminuye. Se estima que cerca de 2 de cada 10 universitarios abandonarían el consumo excesivo episódico de alcohol sin ayuda formal. En otras culturas, este proceso estaría relacionado con situaciones vitales, como por ejemplo, el estado civil [15]. Por otro lado el papel que podrán jugar consecuencias como los problemas psíquicos, episodios de amnesia anterógrada, los problemas físicos o las lesiones no han sido estudiados como factores asociados al abandono de este patrón de consumo. La importancia de incluir estos factores radica en que, si bien se asocian a la severidad del trastorno por uso de alcohol, podría pensarse que tienen un rol relevante en relación al cambio en los patrones de consumo de riesgo.

A pesar de la importancia de estudiar los factores que se relacionarían con el cambio del patrón de consumo, el conocimiento en esta área es una vacante en nuestro contexto. Además de un importante conocimiento teórico, identificar las condiciones en las cuales los jóvenes abandonan el consumo excesivo podría ser de ayuda para diseñar intervenciones comunitarias para reducir el consumo de alcohol [19]. Por ello, este estudio se propone estimar la prevalencia de cambio natural del consumo excesivo episódico del alcohol y explorar su relación con lesiones bajo los efectos del alcohol y problemas de salud física, problemas psíquicos, episodios de amnesia anterógrada, consumo de otras sustancias, género y estado civil, en universitarios que nunca han buscado ayuda por problemas con el alcohol.

\section{Método}

\section{Participantes}

Los datos de este estudio descriptivo transversal provienen de una muestra probabilística aleatoria por conglomerados de 1708 estudiantes de primer a tercer año de distintas carreras de la Universidad Nacional de Mar del Plata. La recolección de datos fue realizada entre abril y noviembre de 2014. La muestra utilizada para este estudio estuvo compuesta por $n=828$ estudiantes que cumplieron los siguientes criterios de selección: ser estudiantes regulares de una carrera de la Universidad Nacional de Mar del Plata, haber presentado alguna vez o presentar actualmente al menos una vez al mes consumo excesivo episódico de alcohol, y nunca haber buscado ayuda por un problema relacionado con el consumo de alcohol. Los datos descriptivos de cada grupo se resumen en la Tabla 1. 


\section{Medidas}

Los posibles sesgos de clasificación fueron controlados mediante el uso de un cuestionario auto-administrado estructurado, se ocultó la hipótesis de trabajo, y se aseguró que no existiera vínculo entre los encargados de la recolección de datos y los participantes.

Bebedores actuales. Se consideró como bebedor actual a todo participante que en los últimos 12 meses hubiese ingerido al menos una bebida con $11 \mathrm{gr}$ de alcohol puro o unidad estándar.

Consumo Excesivo Episódico de Alcohol. Se evaluó mediante el enfoque de cantidadfrecuencia graduada, i. e. preguntas prefijadas sobre la frecuencia de consumo de alcohol en diferentes cantidades de unidades estándar de bebidas alcohólicas [20]. El consumo excesivo episódico pasado se refirió a quienes no consumieron cinco o más unidades estándar de alcohol en una ocasión en los últimos 12 meses, pero sí antes; el actual incluyó a todo aquel que en los últimos 12 meses hubiese consumido cinco o más unidades estándar de alcohol en una ocasión, al menos una vez al mes.

Cambio Natural del Consumo Excesivo Episódico de Alcohol. Se consideró como positivo a todo aquel que informó haber consumido cinco o más unidades estándar de alcohol en una ocasión en el pasado, pero no en los últimos 12 meses, y que no buscó ayuda por un problema relacionado con el consumo de alcohol.

Problemas por consumo de alcohol (lesiones bajo los efectos del alcohol, problemas de salud físicos derivados del consumo de alcohol, problemas psíquicos por el consumo de alcohol y episodios de amnesia anterógrada inducida por el alcohol): Se indagó si la persona había presentado alguna de estas consecuencias del consumo de alcohol alguna vez en la vida (positivo/negativo). Los problemas de salud física (enfermedad de hígado o hepatitis, enfermedad de estómago o sangre al vomitar, hormigueo o entumecimiento en los pies, problemas de memoria aun cuando no estaba bebiendo, pancreatitis) y psíquicos (falta de interés en las actividades acostumbradas, tristeza o depresión, suspicacia o desconfianza y pensamientos extraños) derivados del consumo de alcohol fueron los incluidos en la Clasificación Internacional de Enfermedades, versión 10 [21].

Consumo de otras sustancias psicoactivas: Se evaluó mediante una pregunta ad-hoc, sobre el consumo, en los últimos 12 meses, de cannabis, cocaína, alucinógenos y otras, construyendo posteriormente una variable dicotómica (consumo/no consumo de otras sustancias). Variables socio-demográficas: Género (femenino/masculino), estado civil (soltero/ casado o unido de hecho).

\section{Procedimiento}

Los participantes se reclutaron en las aulas de cursada de las distintas asignaturas, y la administración tuvo lugar en el horario de clase, con una duración de 20 minutos aproximadamente. Estuvieron presentes investigadores para responder eventuales dudas. El proyecto contó con la aprobación al Comité de Ética del Instituto Nacional de Epidemiología. La participación fue voluntaria y anónima, y se obtuvo el consentimiento informado. Se entregó una hoja de información general del estudio, formas de contactar a los responsables e información sobre centros de asistencia de problemas por consumo de alcohol.

\section{Análisis de datos}

Se efectuaron análisis descriptivos para evaluar la prevalencia del cambio natural del consumo excesivo episódico dentro del total de la muestra y dentro de los estudiantes bebedores actuales. Luego, se realizaron análisis bivariados por separado para estimar la probabilidad de haber presentado lesiones bajo los efectos del alcohol, problemas de salud derivados del consumo de alcohol, problemas psicológicos durante el consumo de alcohol y episodios de amnesia anterógrada inducida por el alcohol (valor de referencia $=$ positivo), probabilidad de haber consumido otras sustancias psicoactivas en el último año (valor de referencia $=$ sí) y estado civil (valor de referencia $=$ casado o unido de hecho) 
entre dos poblaciones: quienes presentaban consumo excesivo episódico de alcohol en la actualidad (grupo de referencia, valor $=0$ ) en contraposición a los que lo habían presentado en el pasado (valor $=1$ ). Seguidamente, estos análisis se estratificaron según el género, para evaluar diferencias en las asociaciones de acuerdo a esta variable. Por último, se realizó un modelo de regresión con todas las variables que resultaron asociadas por separado en la muestra total; así, se evaluó el ajuste para la predicción del cambio en el consumo excesivo episódico (grupo de referencia =consumo excesivo episódico actual), con respecto de presentarlo en la actualidad (0). Los análisis se efectuaron con el SPSS 12.0 para Windows.

\section{Resultados}

Del total de los estudiantes (incluidos los abstemios), la prevalencia de cambio natural en el consumo excesivo episódico fue de 7\% (IC 95\%:5-8), mientras que la prevalencia anual de consumo excesivo episódico fue del 53\% (IC 95\%: 51-56); dentro de los bebedores actuales, la prevalencia fue de 5\% (IC 95\%: 4-6) y 60\% (IC 95\%: 57-63) respectivamente (no se muestra en Tablas).

Tabla N $\mathbf{N}^{\mathbf{0}}$ : Características de la Muestra, Estudiantes Universitarios con Consumo Excesivo Episódico de Alcohol Presente y Pasado, N=828, Mar del Plata, 2014.

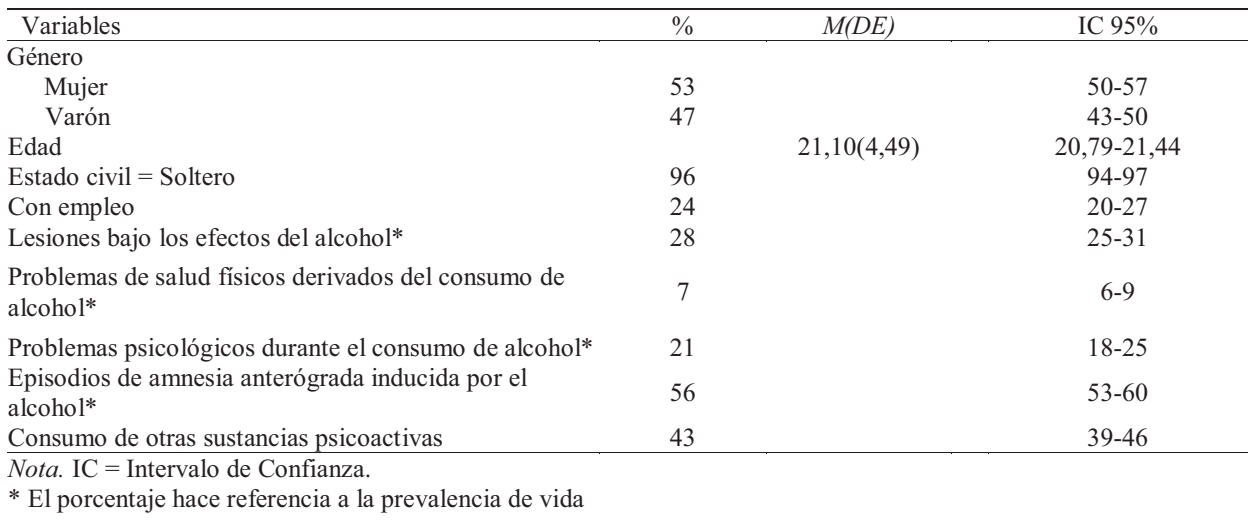

Los análisis de regresión indicaron que la prevalencia de problemas de salud físicos por el consumo de alcohol no fue diferente entre los que presentaron un cambio natural y los que actualmente tenían un consumo excesivo episódico, cuando el resto de las variables consideradas sí lo eran. La consecuencia con menor probabilidad para el grupo de los que cambiaron el consumo excesivo episódico fueron las lesiones bajo los efectos del alcohol (al menos un $10 \%$ menos probable). Por otro lado, la que más variabilidad explicó entre ambos grupos aun en el análisis estratificado fue los episodios de amnesia anterógrada inducidos por el alcohol (aproximadamente el 15\%). En el análisis estratificado según el género se observaron diferencias en algunas asociaciones. El estado civil, que en el total de la muestra indicó que quienes estaban casados tenían casi 5 veces más probabilidad de haber cambiado el consumo excesivo episódico de alcohol, sólo fue significativo para las mujeres. Por su parte, el consumo de otras sustancias psicoactivas, cuya probabilidad en el total de la muestra fue un $33 \%$ menor para los que cambiaron, fue $41 \%$ menos probable en las mujeres y $29 \%$ menos probable en los varones. Inversamente, los problemas psíquicos parecieron ser menos probables en los varones que abandonaron el consumo excesivo episódico (21\%) que en las mujeres que también lo hicieron (Tabla 2).

Tabla N'2: Probabilidad de cambio en el consumo excesivo episódico de alcohol para la muestra total y según el género, Estudiantes Universitarios, N=828, Mar del Plata, 2014 


\begin{tabular}{lrrr}
\hline Variable & $P$ & OR (IC 95\%) & $\mathrm{R}^{2}$ \\
\hline Muestra total & & & \\
$\quad$ Lesiones bajo los efectos del alcohol &, 001 &, $22(, 10-, 47)$ &, 05 \\
$\quad$ Problemas de salud físicos derivados del consumo de alcohol &, 248 &, $54(, 19-1,53)$ &, 00 \\
$\quad$ Problemas psicológicos durante el consumo de alcohol &, 001 &, $11(, 03-, 35)$ &, 07 \\
$\quad$ Episodios de amnesia anterógrada inducida por el alcohol &, 001 &, $13(, 07-, 24)$ &, 15 \\
$\quad$ Consumo de otras sustancias psicoactivas &, 001 &, $33(, 19-, 56)$ &, 05 \\
$\quad$ Estado civil &, 001 & $4,87(2,16-10,98)$ &, 03 \\
Mujeres & & & \\
Lesiones bajo los efectos del alcohol &, 002 &, $22(, 08-, 56)$ &, 06 \\
Problemas de salud físicos derivados del consumo de alcohol &, 808 &, $86(, 25-2,96)$ &, 00 \\
Problemas psicológicos durante el consumo de alcohol &, 004 &, $06(, 01-, 41)$ &, 09 \\
Episodios de amnesia anterógrada inducida por el alcohol &, 001 &, $15(, 07-, 31)$ &, 15 \\
Consumo de otras sustancias psicoactivas &, 013 &, $41(, 20-, 83)$ &, 03 \\
$\quad$ Estado civil &, 003 & $5,32(1,77-15,98)$ &, 03 \\
Varones & & & \\
$\quad$ Lesiones bajo los efectos del alcohol &, 021 &, $24(, 07-, 81)$ &, 04 \\
Problemas de salud físicos derivados del consumo de alcohol &, 238 &, $29(, 04-2,24)$ &, 01 \\
$\quad$ Problemas psicológicos durante el consumo de alcohol &, 034 &, $21(, 05-, 90)$ &, 04 \\
Episodios de amnesia anterógrada inducida por el alcohol &, 001 &, $11(, 04-, 30)$ &, 16 \\
Consumo de otras sustancias psicoactivas &, 005 &, $29(, 12-, 69)$ &, 06 \\
Estado civil &, 083 & $3,31(, 85-12,86)$ &, 01 \\
\hline
\end{tabular}

Nota. Los resultados expresan la probabilidad de responder positivamente a las variables relacionadas con el consumo de alcohol alguna vez en la vida, haber consumido otras sustancias psicoactivas en los últimos 12 meses, y estar casados entre el grupo de los que presentaron consumo excesivo episódico de alcohol en los últimos 12 meses (0) versus los que lo presentaron hace más de 12 meses (1). IC = Intervalo de Confianza.

Finalmente, al incluir las variables asociadas (lesiones, problemas psicológicos y episodios de amnesia anterógrada inducidos por el alcohol, el uso de otras sustancias psicoactivas y el estado civil) en el modelo para estimar su ajuste, se observó que este fue casi perfecto, Hosmer-Lemeshow $=.91$. Las variables en conjunto explicaron un $23 \%$ de la variabilidad entre los que cambiaron el consumo excesivo episódico de alcohol y los que no $R^{2}$ Nagelkerke $=.234$.

\section{Discusión}

El objetivo del presente fue estimar la prevalencia de consumo excesivo episódico del alcohol y cambio natural del mismo, y explorar su relación con consecuencias derivadas del consumo de alcohol, el consumo de otras sustancias, y variables sociodemográficas, en universitarios que nunca han buscado ayuda por problemas con el alcohol. En primer lugar, la prevalencia de cambio natural del consumo excesivo episódico fue inferior a la hallada en otros estudios [15]. Esto podría deberse a variaciones culturales, tales como la gran aceptabilidad del consumo de alcohol en nuestro medio, un hecho que ha sido señalado en estudios de recuperación natural de trastornos por uso de alcohol [22]. La prevalencia de cambio natural entre quienes continuaron bebiendo alcohol en el último año fue levemente menor cuando se excluyeron los abstemios del análisis; esto podría indicar que quienes dejan de lado este patrón de consumo logran mayormente reducir su ingesta a un nivel de menor riesgo, mientras que una proporción menor parece abandonar definitivamente el consumo de alcohol.

Respecto de las variables consideradas, cabe destacar que las lesiones bajo los efectos del alcohol fueron la consecuencia menos probable en quienes cambiaron el consumo de alcohol, algo similar a lo encontrado en la literatura de otros contextos [23]. Esto podría deberse a que el grupo que cambió su consumo de alcohol, también modificó su exposición a situaciones de riesgo físico. O bien, puede plantearse que estas personas tienden a involucrarse en menos conductas de riesgo en general, lo que las diferenciaría de los consumidores excesivos episódicos que persisten en este comportamiento. 
Por otro lado, los episodios de amnesia anterógrada inducida por el alcohol explicaron una alta proporción de la variabilidad entre ambos grupos. Esto es interesante, ya que esta variable ha sido utilizada para validar la severidad de los trastornos por uso de alcohol [16, 17], por lo que este resultado podría indicar que quienes persisten en el consumo excesivo episódico también tienen problemas más severos por el uso de alcohol. Adicionalmente, un estudio en el contexto local ha encontrado que la prevalencia actual de estos episodios depende de haberlos experimentado alguna vez en la vida. Es decir, una vez que se ha padecido un episodio de amnesia anterógrada inducida por el alcohol, se tiene una mayor probabilidad de volver a experimentarlos [24], con el daño neurocognitivo que ello supone [25]. Podría darse el caso de que quienes persisten en este consumo hayan sufrido más daños en este sentido, lo que les dificultaria abandonar el consumo excesivo episódico naturalmente.

Los problemas físicos por uso de alcohol no resultaron asociados al cambio natural de consumo. Esto puede deberse a que en este grupo existe una prevalencia menor de problemas de salud físicos derivados del consumo de alcohol, debido a que el deterioro físico aparece en etapas tardías, cuando el tratamiento por un problema con el uso de alcohol es más difícil [26]. Es decir, es esperable que sólo una pequeña cantidad de estudiantes presenten este problema, afectando la potencia de las asociaciones. Sin embargo, también hay que considerar que se trata de una primera aproximación a un fenómeno poco estudiado en un grupo donde el consumo excesivo está extendido y es muy aceptado [27].

El uso de otras sustancias psicoactivas también indicó una menor probabilidad de pertenecer al grupo que cambió su consumo. Dado el deterioro que produciría el uso de múltiples sustancias [28,29], podría pensarse que estos resultados se relacionan con la menor probabilidad de episodios de amnesia anterógrada; es decir, a menor uso de múltiples sustancias, también es menor la probabilidad de episodios de amnesia anterógrada. Asimismo, respecto de las lesiones, esta información parecería indicar que quienes abandonan el consumo naturalmente tienden a exponerse a conductas de riesgo en menor medida. Determinar si estas personas tienen dificultades para abandonar el consumo excesivo naturalmente debido a daños producidos por el alcohol y el consumo de otras sustancias o bien se relaciona con características pre existentes comunes a ambos (e.g. rasgos de personalidad o una menor autorregulación] es una área en el que se necesitan mayores desarrollos.

Estar casado o unido de hecho disminuyó la probabilidad de presentar consumo excesivo episódico en la actualidad tal como se encontró en otros estudios [15]. No obstante, en el análisis estratificado, sólo se mantuvo en las mujeres. Esto puede deberse a que estas mujeres quizás controlen su consumo al formar una familia y orientarse hacia la crianza [30]. Nuevos estudios de género podrían profundizar esta cuestión.

A pesar de ciertas limitaciones, como el escaso número de personas que realizaron un cambio natural del consumo, hasta donde sabemos, este trabajo presenta por primera vez en nuestro contexto una aproximación al estudio de los cambios de hábitos en salud, en particular del cambio natural del consumo excesivo episódico, un problema de salud pública a nivel mundial [31]. Los resultados presentados plantean a la vez nuevos interrogantes para avanzar en el conocimiento de un campo que, en otros contextos, promete intervenciones de base comunitaria efectivas. Mediante intervenciones simples que incluyen folletos con información y a un bajo costo, podría estimularse el cambio de hábito, el autocontrol sobre el consumo y la búsqueda de ayuda [19]. Los resultados expuestos indicarían que las personas que aún no tuvieron ciertas consecuencias del consumo de alcohol y no consumen otras sustancias psicoactivas, estarían en condiciones de abandonar el consumo excesivo episódico de alcohol sin ayuda formal y con cierta orientación. Sin embargo, los jóvenes con un mayor deterioro se beneficiarían de una intervención de otro tipo. Estas conclusiones permiten pensar en el desarrollo de tecnologías para detectar ambas condiciones. 
En cuanto a nuevas líneas de investigación, sería interesante la comparación de esta población con otras de diferentes características, como la población clínica o adultos de más edad. El estudio de la frecuencia de estos problemas también podría resultar de interés en la identificación de umbrales de eventos que podrían motivar a un cambio en el comportamiento, lo que resultaría en otro avance teórico en el campo.

\section{Agradecimientos}

Este trabajo fue financiado parcialmente por el Consejo Nacional de Investigaciones Científicas y Técnicas, y por la Universidad Nacional de Mar del Plata, Argentina.

\section{Bibliografía}

1. Monteiro MG. Alcohol y salud pública en las Américas: un caso para la acción. Pan American Health Organization; 2007.

2. Bischof G, Rumpf H, Hapke U, Meyer C, John U. Gender differences in natural recovery from alcohol dependence. J Stud Alcohol 2000; 61(6):783-786. doi:10.15288/ jsa.2000.61.783

3. Harford TC, Grant BF, Yi HY, Chen CM. Patterns of DSM-IV alcohol abuse and dependence criteria among adolescents and adults: results from the 2001 National Household Survey on Drug Abuse. Alcohol Clin Exp Res 2005; 29(5):810-828. doi: 10.1097/01.ALC.0000164381.67723.76

4. Karam E, Kypri K, Salamoun M. Alcohol use among college students: an international perspective. Curr Opin Psychiatry 2007; 20(3):213-221. doi: 10.1097/ YCO.0b013e3280fa836c

5. Miguez HA. Cultural patterns of social alcohol use in Buenos Aires' students (Argentina). Vertex 2009; 20(87):325-328.

6. National Institute of Alcohol Abuse and Alcoholism. NIAAA Council approves definition of binge drinking. NIAAA newsletter 2004; 3:3.

7. Dantzer C, Wardle J, Fuller R, Pampalone SZ, Steptoe A. International study of heavy drinking: attitudes and sociodemographic factors in university students. Journal of American College Health 2006; 55(2):83-90. doi: 10.3200/JACH.55.2.83-90

8. Hingson RW, Heeren T, Zakocs RC, Kopstein A, Wechsler H. Magnitude of alcoholrelated mortality and morbidity among US college students ages 18-24. J Stud Alcohol Drugs 2002; 63(2):136. doi: 10.15288/jsa.2002.63.136

9. Schimdt LA. Trends in access to addiction treatment, 1984-2004. Paper presented at the annual meeting of the American Public Health Association, Washington, DC 2007.

10. Caldeira KM, Kasperski SJ, Sharma E, Vincent KB, O'Grady KE, Wish ED, et al. College students rarely seek help despite serious substance use problems. J Subst Abuse Treat 2009; 37(4):368-378. doi: 10.1016/j.jsat.2009.04.005

11. Cremonte M, Conde K, Remaggi, M. Consumo Excesivo Episódico de Alcohol en Estudiantes Universitarios. IV Congreso Marplatense de Psicología. Ideales sociales, psicología y comunidad 2009.

12. Bingham CR, Shope JT, Tang X. Drinking behavior from high school to young adulthood: differences by college education. Alcohol Clin Exp Res 2005 29(12):21702180. doi: 10.1097/01.alc.0000191763.56873.c4

13. Keyes KM, Grant BF, Hasin DS. Evidence for a closing gender gap in alcohol use, abuse, and dependence in the United States population. Drug Alcohol Depend 2008; 93(1-2):21-29. doi: 10.1016/j.drugalcdep.2007.08.017

14. Kim JH, ScD, Chan KWC, BA, Chow JKW, MSocSci, Fung KP, PhD, Fong BYF, MBBS, Cheuk KK, MPhil, et al. University Binge Drinking Patterns and Changes in Patterns of Alcohol Consumption Among Chinese Undergraduates in a Hong Kong University. Journal of American College Health 2009; 58(3):255-265. doi: 


\section{$10.1080 / 07448480903295318$}

15. Vik PW, Cellucci T, Ivers H. Natural reduction of binge drinking among college students. Addict Behav 2003; 28(4):643-655. doi: 10.1016/S0306-4603(01)00281-7

16. Hasin D, Van Rossem R, McCloud S, Endicott J. Alcohol dependence and abuse diagnoses: Validity in community sample heavy drinkers. Alcohol Clin Exp Res 1997; 21(2):213-219. doi: 10.1111/j.1530-0277.1997.tb03752.x

17. Hasin D, Paykin A. Dependence symptoms but no diagnosis: diagnostic orphans' in a community sample. Drug Alcohol Depend 1998; 50(1):19-26. doi:10.1016/S03768716(98)00007-6

18. Kuntsche E, Rehm J, Gmel G. Characteristics of binge drinkers in Europe. Soc Sci Med 2004; 59(1):113-127. doi: 10.1016/j.socscimed.2003.10.009

19. Sobell LC, Sobell MB, Leo GI, Agrawal S, Johnson $\square$ Young L, Cunningham JA. Promoting Self $\square$ Change With Alcohol Abusers: A Community $\square$ Level Mail Intervention Based on Natural Recovery Studies. Alcoholism: Clinical and Experimental Research 2002; 26(6):936-948. doi: 10.1111/j.1530-0277.2002.tb02624.x

20. Gmel G, Graham K, Kuendig H, Kuntsche S. Measuring alcohol consumptionshould the 'graduated frequency'approach become the norm in survey research? Addiction. 2005; 101(1):16-30. doi: 10.1111/j.1360-0443.2005.01224.x

21. World Health Organization. Global strategy to reduce the harmful use of alcohol; 2010.

22. Klingemann H, Sobell MB, Sobell LC. Continuities and changes in self $\square$ change research. Addiction 2010;105(9):1510-1518. doi: 0.1111/j.1360-0443.2009.02770.x

23. Barnett NP, Goldstein AL, Murphy JG, Colby SM, Monti PM. "I'll never drink like that again": characteristics of alcohol-related incidents and predictors of motivation to change in college students. J Stud Alcohol 2006 ;67(5):754-763. doi: 10.15288/ jsa.2006.67.754

24. Conde K, Fischetti MA, Cuesta J, Cremonte M. Alcohol y Uso de Múltiples Sustancias Psicoactivas en Estudiantes de la Universidad Nacional de Mar del Plata. VI Congreso Marplatense de Psicología. La psicología como promotora de derechos. Hacia la interdisciplinariedad de las Prácticas en los escenarios actuales. Mar del Plata, Argentina 2014.

25. Zeigler DW, Wang CC, Yoast RA, Dickinson BD, McCaffree MA, Robinowitz CB, et al. The neurocognitive effects of alcohol on adolescents and college students. Prev Med 2005; 40(1):23-32. doi: 10.1016/j.ypmed.2004.04.044

26. Atkinson RM, Misra S, Ryan SC, Turner JA. Referral paths, patient profiles and treatment adherence of older alcoholic men. J Subst Abuse Treat 2003; 25(1):29-35. doi: 10.1016/S0740-5472(03)00048-5

27. Cicognani E, Zani B. Alcohol Use Among Italian University Students: The Role Of Sensation Seeking, Peer Group Norms and Self-Efficacy. J Alcohol Drug Educ 2011; 55(2):17-36.

28. Midanik, LT, Tam TW, Weisner C. Concurrent and simultaneous drug and alcohol use: results of the 2000 National Alcohol Survey. Drug Alcohol Depend 2007; 90(1):72-80. https://doi.org/10.1016/j.drugalcdep.2007.02.024

29. Pape H, Rossow I, Storvoll EE. Under double influence: assessment of simultaneous alcohol and cannabis use in general youth populations. Drug Alcohol Depend 2009; 101(1):69-73. doi: 10.1016/j.drugalcdep.2008.11.002

30. Nolen-Hoeksema S, Hilt L. Possible contributors to the gender differences in alcohol use and problems. The Journal of general psychology 2006; 133(4): 357-374. doi: 10.3200/GENP.133.4.357-374

31. World Health Organization. Global status report on alcohol and health-2014. World Health Organization; 2014. 